Published in final edited form as:

Biol Psychol. 2012 October ; 91(2): 302-306. doi:10.1016/j.biopsycho.2012.08.002.

\title{
Neural Responses to Infants linked with Behavioral Interactions and Testosterone in Fathers
}

\author{
Patty X. Kuo ${ }^{\mathrm{a}, 1}$, Joshua Carp ${ }^{\mathrm{b}}$, Kathleen C. Light ${ }^{\mathrm{c}}$, and Karen M. Grewen ${ }^{\mathrm{a}, \mathrm{d}}$ \\ Department of Psychology, University of North Carolina \\ Department of Psychology, University of Michigan \\ Department of Anesthesiology, University of Utah \\ Department of Psychiatry, University of North Carolina
}

\begin{abstract}
Few fMRI studies have investigated the brain-behavioral basis of parenting in human fathers. Ten fathers were videotaped and gave salivary testosterone samples while interacting with their 2-4 month old infants, and viewed video clips of their own infant and an unfamiliar age-, ethnicityand sex-matched other infant during an fMRI protocol. Infant stimuli activated a network of prefrontal and subcortical brain regions. Furthermore, a subset of these regions activated significantly more to own (OWN) than other (OTHER) infants. Finally, neural responses to OWN versus OTHER were linked with paternal sensitivity, paternal reciprocity, and testosterone. In sum, our results provide a novel perspective on the links between brain, behavior, and hormones in fathers.
\end{abstract}

\section{Keywords}

fathers; testosterone; fMRI; infant

\begin{abstract}
Previous studies have investigated maternal responses to infant stimuli (Kim et al., 2011; Noriuchi, Kikuchi, \& Senoo, 2008; Strathearn, Li, Fonagy, \& Montague, 2008), but less is known about the neural basis of parenting in fathers. Recent research on fathers showed that own-infant stimuli evoked greater activation than other-infant stimuli throughout a network of brain regions implicated in reward processing, empathy, and memory (Swain, Lorberbaum, Kose, \& Strathearn, 2007). However, these studies relied on static image stimuli. Studies of infant-related responses in mothers suggest that more complex stimuli, such as video clips, may provide a better understanding of the neural correlates of parenting behavior. For example, in mothers, infant videos activate brain regions related to emotion regulation and habitual responses that do not respond to infant photos (Swain, 2008). Furthermore, although hormonal responses play a critical role in paternal behavior (Storey,
\end{abstract}

\footnotetext{
(C) 2012 Elsevier B.V. All rights reserved.

Correspondence concerning this article should be addressed to Patty X. Kuo, CB\# 7175, Medical Wing D, Emergency Room Drive, Chapel Hill, NC USA, 27599-7175.pkuo@email.unc.edu.

${ }^{1}$ Present address: Patty X. Kuo, East Hall 4027, 530 Church Street, Ann Arbor, MI 48109. pkuo@umich.edu

Patty X. Kuo is now at Department of Psychology, University of Michigan.

Publisher's Disclaimer: This is a PDF file of an unedited manuscript that has been accepted for publication. As a service to our customers we are providing this early version of the manuscript. The manuscript will undergo copyediting, typesetting, and review of the resulting proof before it is published in its final citable form. Please note that during the production process errors may be discovered which could affect the content, and all legal disclaimers that apply to the journal pertain.
} 
Walsh, Quinton, \& Wynne-Edwards, 2000), previous studies have not examined the relationship between hormones and infant-related neural activation.

One hormone of particular interest in paternal behavior is testosterone. The Challenge Hypothesis has been proposed as an evolutionary tradeoff between the costs and benefits of males maintaining high testosterone across various reproductive behaviors: seeking new mates and sexual activity are characterized by high testosterone, while parenting effort decreases with elevations in testosterone (Wingfield, Hegner, Dufty Jr, \& Ball, 1990). As such, an environment where available mates are scarce and offspring are dependent would promote a parenting effort strategy characterized by low testosterone, often seen in humans. Fatherhood decreases testosterone levels in men longitudinally (Gettler, McDade, Feranil, \& Kuzawa, 2011), and fathers have lower testosterone levels than non-fathers (Gray, Parkin, \& Samms-Vaughan, 2007; Kuzawa, Gettler, Muller, McDade, \& Feranil, 2009). Findings from animal literature also reflect lowered testosterone in parous mammals (Wynne-Edwards \& Reburn, 2000).

Moreover, parenting behaviors play a significant role in modulating testosterone. Fathers who are involved in at least three hours of child caregiving have lower testosterone levels than fathers who are not involved (Gettler, et al., 2011), suggesting that it is not only parenting status that influences testosterone levels, but also parenting behaviors. This phenomenon has also been shown in experimental settings. Decreases in men's testosterone levels in response to caregiving behaviors have been found using infant dolls (van Anders, Tolman, \& Volling, 2012). Given that testosterone is responsive to both parenting status and, more importantly, parenting behavior, we investigated the role of testosterone in this study. Administered testosterone increases activation in the thalamocingulate region in response to infant cries in women (Bos, Hermans, Montoya, Ramsey, \& van Honk, 2010), lending evidence for the interconnections between testosterone and neural circuits relevant to parenting. However, no previous research has investigated links between endogenous testosterone and neural responses to parenting-relevant stimuli.

Thus, the present study investigated neural responses to dynamic displays of infants in fathers, as well as the relationships between neural activity, testosterone, and parental behavior.

\section{Method}

Participants were 10 right-handed fathers, (mean age: 33.9; range: 28-44 yrs.) and their infants (8-19 weeks old, 5 female) living in the Southeastern United States. All fathers were in a co-habiting marital relationship with the infant's mother. The sample was fairly diverse. Fathers' ethnic/racial background was as follows: six White/Caucasian, two Asian/AsianAmerican, one Black/African-American and one Latino/Hispanic father. All fathers reported completing at least some college education. Fathers' reported total household income ranged from $\$ 30,000$ to $\$ 79,999$, with 5 of the fathers reporting a total household income of at least $\$ 60,000$.

Infant interaction video was missing for two fathers, and hormone data was missing for one father. Thus, analyses with hormone data were conducted with nine fathers, and analyses with infant interaction data were conducted with eight fathers. All 10 fathers participated in the fMRI paradigm.

Fathers and their infants attended the initial lab visit, and informed consent was received from fathers for both their participation and their infants' participation. Fathers provided salivary testosterone samples and questionnaire data; fathers and infants engaged in a videotaped interaction during this visit. The visit was conducted between 1200 and 1700 hours to 
minimize circadian variability. Unstimulated saliva samples for testosterone were collected after a 5-min baseline and 5-min videotaped face-to-face infant interaction. During the infant interaction period, infants were seated in a carrier and fathers were instructed to act as they normally would with their baby. Saliva samples were assayed for testosterone in duplicate using a Salimetrics ELISA kit with an intra-assay CV of 2.5\% and inter-assay CV of 5.6\%. Paternal behavior during videotaped interactions was independently coded by blinded raters for Parental Sensitivity (PS) and Parental Reciprocity (PR). PS, adapted from the NICHD Maternal Sensitivity Scale (NICHD Early Child Care Research Network, 1999 \& 2000), focuses on the parent showing awareness of the child's mood, needs, interests, and capabilities, and observing the child's cues to allow the child to determine the pace and activities of the interaction. PR measures the parent's reciprocal interaction with the infant, including the following typical items: "Waits for infant to initiate interactions" and "Mirrors infant's facial expression and emotions", and was adapted from the Mother Infant Interaction During Feeding Scale (Chatoor et al., 1997) by one of the authors, (K.C.L.).

To acquire stimuli for the fMRI paradigm, infants were video-taped separately sitting in an infant carrier alone for five minutes. Only video segments of infants showing either neutral or slight positive affect (smiling) were chosen to be used as video stimuli. None of the 15 second segments showed any strong positive affect or smiles that lasted for the entire segment. Any smiles shown in the segments were fleeting and the segments can be mostly characterized as neutral. Baseline control stimuli were acquired by videotaping a Raggedy Andy doll placed in an infant carrier for five minutes. The baseline control condition moved slightly with swinging in the infant carrier to emulate the infants' slight movements.

Fathers attended the second lab visit within four weeks of the initial lab visit to provide imaging data. Upon giving consent, fathers filled out a screening questionnaire to assess any permanent metal in the body and were instructed to lie supine in the fMRI scanner. Fathers were asked to watch the video clips presented with attention.

\section{Imaging acquisition}

Images were acquired with a 3T Siemens head-only MRI scanner (Allegra, Siemens Medical System, Erlangen, Germany). Functional images were acquired using T2*-weighted echo planar imaging (TR=1500 ms, TE=30 ms, 28 slices, resolution $4 \times 4 \times 4 \mathrm{~mm}$ ). High-resolution anatomical scans were collected for spatial normalization of the functional data (MPRAGE; $\mathrm{TR}=1750 \mathrm{~ms}, \mathrm{TE}=4.38 \mathrm{~ms}, \mathrm{TI}=900 \mathrm{~ms}, 144$ slices, resolution $1 \times 1 \times 1 \mathrm{~mm}$ ).

\section{Imaging Stimuli}

Five-minute digital video recordings of neutral or smiling infants, acquired during the lab visit, were used as stimuli during the fMRI visit. Each father was presented with 15-second video segments of his own infant (OWN), a sex-, age-, and ethnicity-matched other infant (OTHER), and a Raggedy Andy doll (DOLL) presented in random order, with no infant clip presented more than once. OTHER stimuli were obtained from either infants of other fathers in this study or infants of mothers in one of the author's (K.M.G.) other study. OTHER infants were chosen by evaluating the age, sex, and ethnicity of the target (OWN) infant and finding another infant who matched OWN by age, sex and ethnicity. Participants completed four 4.5 min runs of this task separated by 30 -second rest periods.

\section{Imaging analysis}

Data were analyzed using SPM8 (Wellcome Department of Cognitive Neurology, London, UK). Images were corrected for slice timing, realigned, co-registered to the structural image, normalized to MNI space, and spatially smoothed (FWHM $8 \mathrm{~mm}$ ). 
Data were modeled as a block design using the general linear model as implemented in SPM8. Boxcar functions for OWN and OTHER were convolved with a synthetic hemodynamic response function. DOLL was treated as a baseline control. Twenty-four head motion regressors were modeled as nuisance covariates, including the linear, squared, timeshifted, and squared time-shifted transformations of the six rigid-body movement parameters. Data were high-pass filtered at $1 / 128 \mathrm{~Hz}$; serial autocorrelations were corrected using AR(1) modeling.

Group-level analyses of whole-brain responses were conducted using a regression model treating subjects as random effects; contrasts were evaluated using one-sample t-tests. Whole-brain analyses used a height threshold of $p<0.005$ and an extent threshold of $\mathrm{k}>=$ 175 voxels. Monte Carlo simulations using the Resting-State fMRI Data Analysis Toolkit (REST, Song Xiao-Wei et al., http://www.restfmri.net) showed that these thresholds yielded a corrected cluster-wise false positive rate of $p<0.05$. This empirical threshold derivation procedure maintains precise control of the cluster-wise false positive rate and increases sensitivity up to fivefold relative to methods that rely solely on voxel-level thresholds (Forman et al., 1995). Region-of-interest (ROI) analyses averaged parameter estimates across $6 \mathrm{~mm}$-radius spheres centered on coordinates of interest. Contrasts between conditions were evaluated using one-sample t-tests; relationships between ROI activation and individual difference measures were assessed using Pearson correlation.

\section{Results}

First, we compared neural responses to the BABY condition (average of OWN and OTHER) and the DOLL condition. Whole-brain analysis of the BABY versus DOLL contrast revealed widespread cortical and subcortical activation (Table 1; Figure 1). The BABY condition evoked greater activation than DOLL in the orbitofrontal cortex, bilateral caudate, bilateral superior parietal lobules, bilateral middle temporal lobes, and a network of lateral frontal regions. DOLL yielded greater activation than the BABY condition in the middle cingulate gyrus, bilateral poster cingulate cortex, and bilateral superior temporal gyrus.

Next, we compared neural responses to the OWN and OTHER conditions. Whole-brain analysis of OWN versus OTHER revealed increased activation for OWN in bilateral inferior frontal gyrus, bilateral supramarginal gyrus, and bilateral middle temporal gyrus; the OTHER condition elicited increased activation in bilateral fusiform gyrus and right striate cortex (Table 2; Figure 2). Whole-brain analysis requires the use of stringent corrections for multiple comparisons that may mask small but significant effects. In contrast, region of interest (ROI) analyses do not require the use of such thresholds and are therefore offer improved sensitivity (Poldrack, 2007; Saxe, Brett, \& Kanwisher, 2006). Thus, we also investigated activation in ROIs defined using the peak coordinates from the BABY versus DOLL contrast (Table 1) and from a study of maternal fMRI responses to infants and adults (Nitschke et al., 2004). This analysis revealed greater activation to OWN than OTHER (Table 3 ) in the right superior frontal gyrus (rSFG), right inferior frontal gyrus (rIFG), right caudate $(\mathrm{rC})$, left caudate $(\mathrm{lC})$, and right orbitofrontal cortex (rOFC).

Finally, we extracted parameter estimates for the OWN versus OTHER contrast from the ROIs listed above and assessed two-tailed Pearson correlations with PR, PS, and testosterone. Activation in rOFC was negatively correlated with both PS $(r=-0.714, p<$. $05)$ and PR $(r=-0.774, p<.05)$. Testosterone following infant interaction was positively correlated with $1 \mathrm{C}$ activation $(r=0.679, p<.05)$. 


\section{Discussion}

We report a novel investigation of the relationships among parenting behavior, testosterone, and neural reactivity to infant stimuli in fathers. In contrast to previous studies, we used video clips of infants rather than static images. We found that BABY videos activated a widespread network of prefrontal and subcortical brain regions when compared to DOLL. Therefore, there may be a network of regions that are more attuned to processing infant faces. Moreover, OWN activated a subset of these regions significantly more than OTHER, and these regions may be important for recognizing and processing OWN infant cues. Finally, we observed that OWN > OTHER responses were linked with paternal sensitivity, reciprocal behavior with infant, and testosterone.

Our results show both commonalities with and differences from previous studies of infantrelated neural activation in parents.

Studies of mothers have reported activation to infant photos in the striatum, medial prefrontal cortex, occipital cortex, insula, ACC, and in some cases amygdala and parahippocampus (Bartels \& Zeki, 2004; Leibenluft, Gobbini, Harrison, \& Haxby, 2004; Nitschke, et al., 2004; Strathearn, et al., 2008). In mothers, silent infant video clips evoke activation in OFC, periaqueductal gray, anterior insula, putamen (Noriuchi, et al., 2008), right anterior temporal pole, left amygdala and visual cortex (Ranote et al., 2004).

Consistent with these results, we found infant-related activation in OFC. We also observed infant-related activation in bilateral caudate, likely reflecting the motivational significance of infant stimuli. Finally, we found that infant stimuli activated a network of frontal and parietal regions involved in attentional control, possibly reflecting heightened attention (Dosenbach, Fair, Cohen, Schlaggar, \& Petersen, 2008). To further investigate gender differences in infant-related neural activity, future studies should directly compare the neural correlates of parenting between mothers and fathers.

Fathers with greater PR and PS showed less OWN > OTHER activation in the rOFC, which may reflect generalized interest in infants. In other words, fathers with high PR and PS may be highly interested in both their own infants and other infants, leading to a reduction in differentiation between OWN- and OTHER-related activation. Alternatively, given that the right OFC is important for tracking changing emotions (Goodkind et al., 2012), fathers with higher PR and PS may have found it more challenging to track the emotions of an unknown infant, and more readily recognized the subtle cues from their own infant. Finally, otherinfant clips may have activated a more anterior "abstract processing" region of the OFC (Sescousse, Redouté, \& Dreher, 2010), which responded to an unknown representation of an infant. Since the orbitofrontal cortex is involved in reward processing and emotion (Kringelbach \& Rolls, 2004), could reflect fathers differentiating between other and own infants.

Previous neuroimaging research on mothers has found that own infant viewing elicited increased activations in the anterior part of the OFC (Minagawa-Kawai et al., 2009), which is inconsistent with our findings. Given that the right OFC is important for tracking changing emotions (Goodkind et al., 2012), it may be that the fathers in our study found it more challenging to track the emotions of an unknown infant, and more readily recognized the subtle cues from their own infant. Alternatively, OTHER may have activated a more anterior "abstract processing" section (Sescousse, Redouté, \& Dreher, 2010) of the OFC which responded to an unknown representation of an infant.

Fathers who had greater testosterone following infant interaction had greater activation to OWN vs. OTHER in $1 \mathrm{C}$, a region linked with approach and emotional behavior (Villablanca, 2010). Happy infant faces also activate the left caudate, indicating that seeing happy infant 
faces is rewarding (Montoya et al., 2012). Fathers who had greater activation to their own infant in this reward region also had higher testosterone after interacting with their infants. The only other study which has investigated testosterone with fMRI and infant stimuli had also found a positive correlation between testosterone and neural response (Bos, et al., 2010), and as such testosterone may play an important role in infant-elicited neural activity. Although high testosterone has been largely negatively correlated with paternal behavior, and levels decrease when high testosterone fathers interact more with their infants (Storey, Noseworthy, Delahunty, Halfyard, \& McKay, 2011), van Anders et al. have suggested that testosterone increases may occur in response to urgent infant stimuli such as cries, and may be linked to parental protective behaviors (van Anders, Goldey, \& Kuo, 2011). Much more research is needed to determine the links between neural activity, testosterone and parenting behaviors.

There are several limitations to this study, including a small sample size, incomplete data for all fathers, and lack of correction for multiple correlations between the imaging and the behavioral and hormonal data, all of which leads to low statistical power. Because of the low statistical power of this experiment, we did not correct significance estimates for multiple comparisons. As such, this study should be replicated with a larger sample. However, while stringent threshold standards in fMRI studies are necessary to avoid type I errors, these same standards can often mask real effects with Type II errors, particularly in social neuroscience studies (Lieberman \& Cunningham, 2009). We did not ask fathers about parity; future research should include this measure. Future research should also include a better control stimulus during the imaging component, such as a fixation cross.

Our results raise important questions about the neural basis of paternal behavior. For example, the present study assessed testosterone levels and neural responses during separate visits; future studies should investigate simultaneous changes in hormones and neural activation while fathers view infant stimuli. Future research might also explore the relationship between fathers' parenting styles and neural responses to infants. In conclusion, our research forms a foundation to explore the biological and behavioral bases of fatherinfant attachment, development of parenting style, and prevention of child neglect or abuse.

\section{Acknowledgments}

The authors thank Social Neuroscience and Psychophysiology Laboratory staff, the UNC Biomedical Research Imaging Center, Joshua Bizzell for fMRI analysis consultation, Megan Muir, Wendy Birmingham and Michaella Lawson for video coding, Cheryl Walker for endocrine assays, and Kai Cortina for statistical analysis consultation. This research was funded by NIH grants K01DA019949 P01DA022446(KG), R01HL084222 (KL), RR00046, and UNC Summer Undergraduate Research Fellowship (PK).

\section{References}

Bartels A, Zeki S. The neural correlates of maternal and romantic love. NeuroImage. 2004; 21(3): 1155-1166. [PubMed: 15006682]

Bos PA, Hermans EJ, Montoya ER, Ramsey NF, van Honk J. Testosterone administration modulates neural responses to crying infants in young females. Psychoneuroendocrinology. 2010; 35(1):114121. [PubMed: 19819079]

Chatoor I, Getson P, Menvielle E, Brasseaux C, O'Donnell R, Rivera Y, et al. A feeding scale for research and clinical practice to assess mother-infant interactions in the first three years of life. Infant Mental Health Journal. 1997; 18(1):76-91.

Dosenbach N, Fair D, Cohen A, Schlaggar B, Petersen S. A dual-networks architecture of top-down control. Trends in Cognitive Sciences. 2008; 12(3):99-105. [PubMed: 18262825] 
Forman SD, Cohen JD, Fitzgerald M, Eddy WF, Mintun MA, Noll DC. Improved assessment of significant activation in functional magnetic resonance imaging (fMRI): Use of a cluster-size threshold. Magnetic Resonance in Medicine. 1995; 33(5):636-647. [PubMed: 7596267]

Gettler LT, McDade TW, Feranil AB, Kuzawa CW. Longitudinal evidence that fatherhood decreases testosterone in human males. Proceedings of the National Academy of Sciences. 2011; 108(39): 16194-16199.

Goodkind MS, Sollberger M, Gyurak A, Rosen HJ, Rankin KP, Miller B, et al. Tracking emotional valence: The role of the orbitofrontal cortex. Human Brain Mapping. 2012; 33(4):753-762. [PubMed: 21425397]

Gray PB, Parkin JC, Samms-Vaughan ME. Hormonal correlates of human paternal interactions: A hospital-based investigation in urban Jamaica. Hormones and Behavior. 2007; 52(4):499-507. [PubMed: 17716675]

Kim P, Feldman R, Mayes LC, Eicher V, Thompson N, Leckman JF, et al. Breastfeeding, brain activation to own infant cry, and maternal sensitivity. Journal of Child Psychology and Psychiatry. 2011; 52(8):907-915. [PubMed: 21501165]

Kringelbach ML, Rolls ET. The functional neuroanatomy of the human orbitofrontal cortex: evidence from neuroimaging and neuropsychology. Progress in Neurobiology. 2004; 72(5):341-372. [PubMed: 15157726]

Kuzawa CW, Gettler LT, Muller MN, McDade TW, Feranil AB. Fatherhood, pairbonding and testosterone in the philippines. Hormones and Behavior. 2009

Leibenluft E, Gobbini MI, Harrison T, Haxby JV. Mothers' neural activation in response to pictures of their children and other children. Biological Psychiatry. 2004; 56(4):225-232. [PubMed: 15312809]

Lieberman MD, Cunningham WA. Type I and Type II error concerns in fMRI research: re-balancing the scale. Social Cognitive and Affective Neuroscience. 2009; 4(4):423-428. [PubMed: 20035017]

Minagawa-Kawai Y, Matsuoka S, Dan I, Naoi N, Nakamura K, Kojima S. Prefrontal Activation Associated with Social Attachment: Facial-Emotion Recognition in Mothers and Infants. Cerebral Cortex. 2009; 19(2):284-292. [PubMed: 18515298]

Montoya JL, Landi N, Kober H, Worhunsky PD, Rutherford HJV, Mencl WE, et al. Regional Brain Responses in Nulliparous Women to Emotional Infant Stimuli. PLoS ONE. 2012; 7(5):e36270. [PubMed: 22590530]

Nitschke JB, Nelson EE, Rusch BD, Fox AS, Oakes TR, Davidson RJ. Orbitofrontal cortex tracks positive mood in mothers viewing pictures of their newborn infants. NeuroImage. 2004; 21(2): 583-592. [PubMed: 14980560]

Noriuchi M, Kikuchi Y, Senoo A. The functional neuroanatomy of maternal love: Mother's response to infant's attachment behaviors. Biological Psychiatry. 2008; 63(4):415-423. [PubMed: 17686467]

Poldrack RA. Region of interest analysis for fMRI. Social Cognitive and Affective Neuroscience. 2007; 2(1):67-70. [PubMed: 18985121]

Ranote S, Elliott R, Abel KM, Mitchell R, Deakin JFW, Appleby L. The neural basis of maternal responsiveness to infants: An fMRI study. NeuroReport: For Rapid Communication of Neuroscience Research. 2004; 15(11):1825-1829.

Saxe R, Brett M, Kanwisher N. Divide and conquer: A defense of functional localizers. NeuroImage. 2006; 30(4):1088-1096. [PubMed: 16635578]

Sescousse G, Redouté J, Dreher J-C. The architecture of reward value coding in the human orbitofrontal cortex. The Journal of neuroscience : the official journal of the Society for Neuroscience. 2010; 30(39):13095-13104. [PubMed: 20881127]

Storey AE, Noseworthy DE, Delahunty KM, Halfyard SJ, McKay DW. The effects of social context on the hormonal and behavioral responsiveness of human fathers. Hormones and Behavior. 2011; 60(4):353-361. [PubMed: 21767539]

Storey AE, Walsh CJ, Quinton RL, Wynne-Edwards KE. Hormonal correlates of paternal responsiveness in new and expectant fathers. Evolution and Human Behavior. 2000; 21(2):79-95. [PubMed: 10785345]

Strathearn L, Li J, Fonagy P, Montague PR. What's in a smile? Maternal brain responses to infant facial cues. Pediatrics (Evanston). 2008; 122(1):40-51. 
Swain JE. Baby stimuli and the parent brain: Functional neuroimaging of the neural substrates of parent-infant attachment. Psychiatry. 2008; 5(8):28-36. [PubMed: 19727273]

Swain JE, Lorberbaum JP, Kose S, Strathearn L. Brain basis of early parent-infant interactions: Psychology, physiology, and in vivo functional neuroimaging studies. Journal of Child Psychology and Psychiatry. 2007; 48(3):262-287. [PubMed: 17355399]

van Anders SM, Goldey KL, Kuo PX. The Steroid/Peptide Theory of Social Bonds: Integrating testosterone and peptide responses for classifying social behavioral contexts.

Psychoneuroendocrinology. 2011; 36(9):1265-1275. [PubMed: 21724336]

van Anders SM, Tolman RM, Volling BL. Baby cries and nurturance affect testosterone in men. Hormones and Behavior. 2012; 61(1):31-36. [PubMed: 22001872]

Villablanca JR. Why do we have a caudate nucleus? Acta Neurobiologiae Experimentalis. 2010; 70(1): 95-105. [PubMed: 20407491]

Wingfield JC, Hegner RE, Dufty AM Jr, Ball GF. The "challenge hypothesis': theoretical implications for patterns of testosterone secretion, mating systems, and breeding strategies. American Naturalist. 1990; 136(6):829-846.

Wynne-Edwards KE, Reburn CJ. Behavioral endocrinology of mammalian fatherhood. Trends in Ecology \& Evolution. 2000; 15(11):464-468. [PubMed: 11050350] 


\section{$\mathrm{BABY}>\mathrm{DOLL}$}

\section{Left Hemisphere}

Right Hemisphere

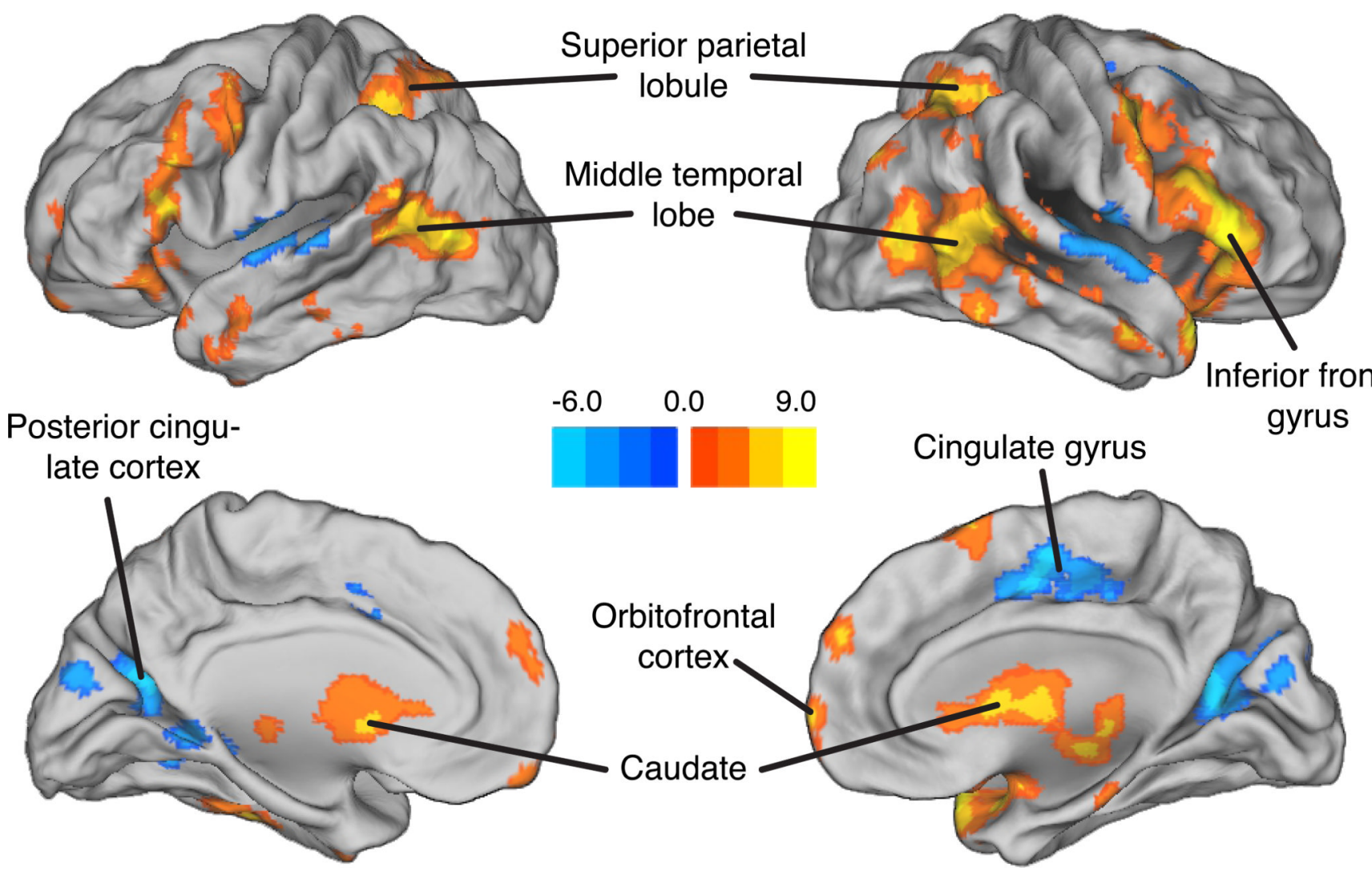

Figure 1.

Brain regions showing greater responses to baby stimuli than DOLL stimuli. Peak activation coordinates and statistics are provided in Table 1. 


\section{OWN > OTHER}

Left Hemisphere

Right Hemisphere
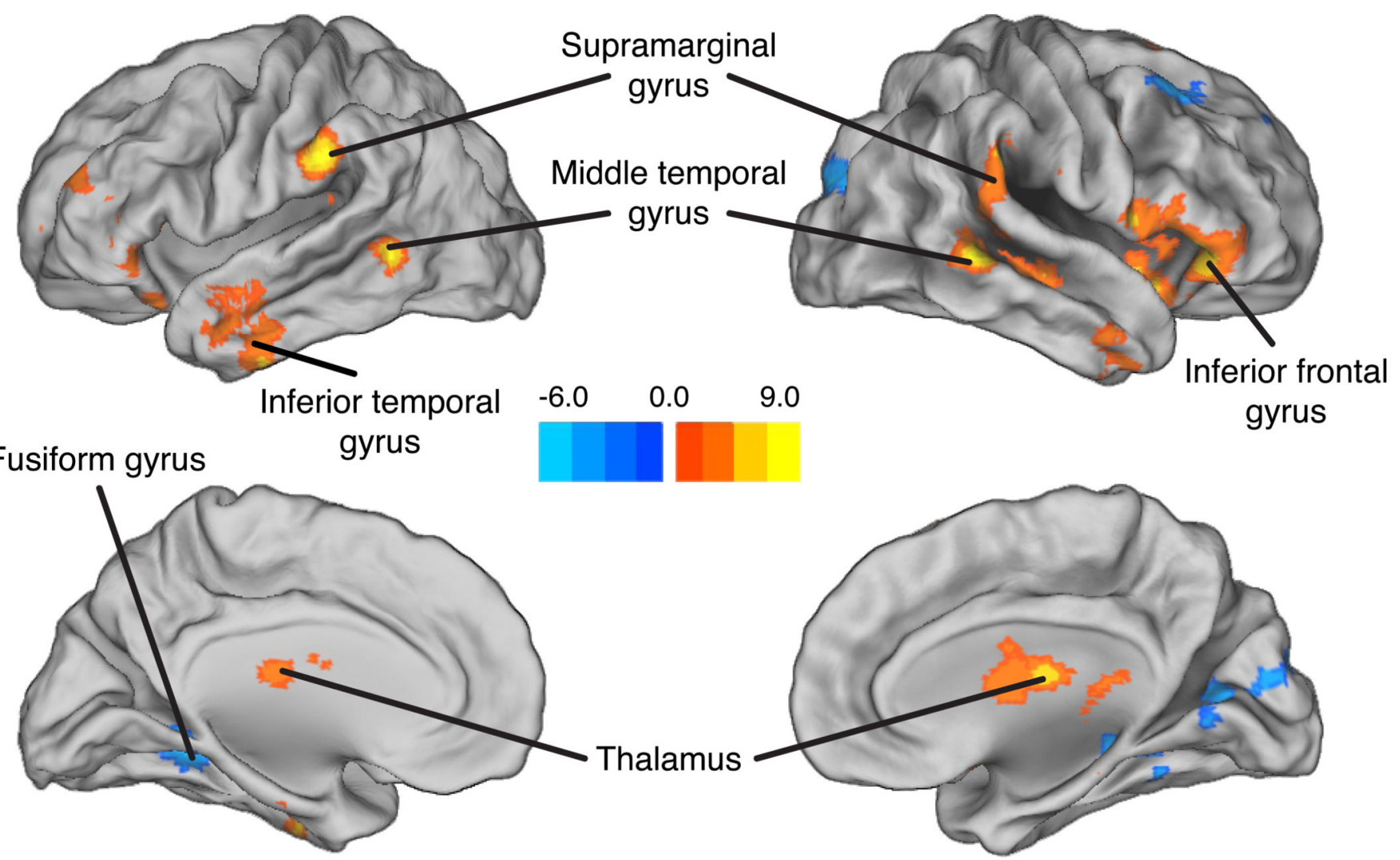

Figure 2.

Brain regions showing greater responses to OWN than OTHER. Peak activation coordinates and statistics are provided in Table 2 . 
Table 1

Neural responses to the BABY versus DOLL contrast.

\begin{tabular}{|c|c|c|c|c|}
\hline \multirow{2}{*}{ Region } & \multicolumn{3}{|c|}{ MNI Coordinates } & \multirow{2}{*}{ Peak t-value } \\
\cline { 2 - 4 } & $\mathbf{X}$ & $\mathbf{Y}$ & $\mathbf{Z}$ & \\
\hline \multicolumn{5}{|c|}{$D O L L$} \\
\hline Orbitofrontal cortex & 2 & 64 & 0 & 5.81 \\
\hline L. superior frontal gyrus & -46 & -2 & 50 & 6.86 \\
\hline R. superior frontal gyrus & 54 & 6 & 50 & 7.88 \\
\hline R. middle frontal gyrus & 52 & 26 & 24 & 9.74 \\
\hline R. inferior frontal gyrus & 48 & 28 & 6 & 9.86 \\
\hline L. caudate & -12 & 2 & 13 & 7.36 \\
\hline R. caudate & -12 & -2 & 18 & 6.23 \\
\hline L. superior parietal lobule & -28 & -68 & 60 & 8.64 \\
\hline R. superior parietal lobule & 36 & -70 & 50 & 6.41 \\
\hline L. middle temporal lobe & -54 & -68 & 8 & 8.21 \\
\hline R. middle temporal lobe & 46 & -74 & 6 & 7.42 \\
\hline \multicolumn{1}{|c|}{$D O L L>B A B Y$} \\
\hline Cingulate gyrus & 6 & -6 & 46 & 5.63 \\
\hline L. superior temporal gyrus & -52 & -12 & 6 & 6.91 \\
\hline R. superior temporal gyrus & 70 & -12 & 2 & 5.73 \\
\hline L. posterior cingulate & -22 & -66 & 16 & 5.38 \\
\hline R. posterior cingulate & 16 & -62 & 12 & 5.85 \\
\hline
\end{tabular}


Table 2

Neural responses to OWN versus OTHER contrast.

\begin{tabular}{|c|c|c|c|c|}
\hline \multirow{2}{*}{ Region } & \multicolumn{3}{|c|}{ MNI Coordinates } & \multirow{2}{*}{ Peak $\boldsymbol{t}$-value } \\
\cline { 2 - 4 } & $\mathbf{X}$ & $\mathbf{Y}$ & $\mathbf{Z}$ & \\
\hline \multicolumn{4}{|c|}{ OTHER } \\
\hline L. inferior frontal gyrus & -44 & 30 & 0 & 4.90 \\
\hline R. inferior frontal gyrus & 42 & 24 & 0 & 7.16 \\
\hline L. supramarginalgyrus & -60 & -32 & 30 & 10.08 \\
\hline R. supramarginalgyrus & 70 & -20 & 32 & 7.67 \\
\hline L. middle temporal gyrus & -56 & -54 & -2 & 8.22 \\
\hline R. middle temporal gyrus & 60 & -42 & 2 & 12.76 \\
\hline & OTHER $>$ OWN \\
\hline L. fusiform gyrus & -28 & -50 & -4 & 6.02 \\
\hline R. fusiform gyrus & 24 & -40 & -4 & 4.57 \\
\hline R. striate cortex & 12 & -66 & 8 & 4.70 \\
\hline
\end{tabular}


Table 3

ROI analyses for OWN versus OTHER contrast.

\begin{tabular}{|c|c|c|c|c|}
\hline \multirow{2}{*}{ Region } & \multicolumn{3}{|c|}{ MNI Coordinates } & \multirow{2}{*}{ t-value } \\
\cline { 2 - 4 } & $\mathbf{X}$ & $\mathbf{Y}$ & $\mathbf{Z}$ & \\
\hline R. superior frontal gyrus & 54 & 6 & 50 & 4.13 \\
\hline R. inferior frontal gyrus & 48 & 28 & 6 & 3.90 \\
\hline R. Caudate & -12 & 2 & 14 & 2.42 \\
\hline L.Caudate & 12 & -2 & 18 & 2.88 \\
\hline & \multicolumn{3}{|c|}{ Tal. Coordinates } & \\
\hline Region & $\mathrm{X}$ & $\mathrm{Y}$ & $\mathrm{Z}$ & $t$-value \\
\hline R. Orbital frontal cortex 1 & 44 & 32 & -15 & 3.57 \\
\hline R. Orbital frontal cortex 2 & 34 & 31 & -10 & 2.51 \\
\hline
\end{tabular}

